\title{
Cerebrospinal fluid drainage in thoracic endovascular aortic repair: mandatory access but tailored placement
}

\author{
Cenea Kemp ${ }^{1,2}$, Yuki Ikeno ${ }^{1}$, Muhammad Aftab $^{1}$, T. Brett Reece ${ }^{1}$ \\ ${ }^{1}$ Division of Cardiothoracic Surgery, Department of Surgery, University of Colorado Anschutz Medical Center, Aurora, CO, USA; ${ }^{2}$ Department of \\ Surgery, University of Colorado Anschutz Medical Center, Aurora, CO, USA \\ Correspondence to: T. Brett Reece, MD. Professor in Cardiothoracic Surgery, Division of Cardiothoracic Surgery, Department of Surgery, \\ University of Colorado School of Medicine/Anschutz Medical Center, 12631 E. 17th Avenue, MS C-302, Aurora, CO 80045, USA. \\ Email: Brett.Reece@cuanschutz.edu.
}

Submitted Apr 27, 2021. Accepted for publication Oct 19, 2021.

doi: 10.21037/acs-2021-taes-12

View this article at: https://dx.doi.org/10.21037/acs-2021-taes-12

\section{Introduction}

The employment of thoracic endovascular aortic repair (TEVAR) for the treatment of thoracic and thoracoabdominal aortic pathology, including both aneurysm and dissection, has greatly improved outcomes for these pathologies. With the increased use of TEVAR, the morbidity and mortality associated with aortic repair has been reduced, though spinal cord ischemia (SCI) resulting in paraplegia and paraparesis remains a significant concern for patients and surgeons. The risk of SCI was previously reported to be as high as $20 \%$ in open aortic aneurysm repairs $(1,2)$. With the introduction of and advancements in endovascular techniques, the risk for SCI following TEVAR has been reported to be $1.5-13 \%(3-5)$. The goal for aortic surgeons remains to eliminate this risk if possible.

The ongoing incidence of SCI in TEVAR is related to coverage of the intercostal arteries and collaterals by the implanted graft, increasing with greater extent of aortic coverage (6). Multiple perioperative adjuncts have been implemented to reduce the risk of SCI in endovascular aortic operations including collateral preservation with subclavian bypass, staged procedures to limit the extent of coverage during any given operation, careful maintenance of intraoperative and postoperative blood pressure and intraoperative neuromonitoring. Multiple pharmaceutical adjuncts have also been presented, though without standardized use amongst institutions. Finally, placement of spinal drains to drain the cerebrospinal fluid (CSF) has been shown to reduce the incidence of SCI through the preservation of spinal perfusion pressure in open and endovascular aortic repairs $(5,7)$. However, CSF drainage has its complications, including but not limited to headache, peri-cordal hematoma, subdural hematoma and even herniation. Concurrently, not all patients undergoing drain placement need them or even have CSF removed once the drains are placed. This led to more restrictive utilization of CSF drains in patients. The preemptive placement of spinal drains in patients prior to undergoing TEVAR remains a contentious topic with no clearly stated guidelines for surgeons to follow.

\section{The importance of SCl protocols}

Multiple institution-specific protocols have been published with the aim of creating a global standardized practice to reduce the incidence of SCI in TEVAR $(4,8)$. These protocols frequently employ the use of permissive hypertension with goals of maintaining the mean arterial pressure (MAP) above a designated point, usually greater than $90 \mathrm{mmHg}$, intraoperative hypothermia, blood transfusion with a hemoglobin goal of $\geq 10 \mathrm{~g} / \mathrm{dL}$ and pharmacologic adjuncts. These protocols also frequently include the use of spinal drains, either preemptively placed in every patient, on a case-by-case basis for those who are deemed to be high risk for SCI preoperatively, or in patients demonstrating symptoms of spinal cord malperfusion at presentation. There are consistent demonstrations of favorable outcomes with regards to SCI $(4,6,8)$ among the various protocols. The effectiveness is multifactorial, owing to a combination of adjuncts that are known to reduce the incidence of SCI rather than any single intervention. 
At our institution, preoperative spinal drains are not routinely placed prior to TEVAR. However, intraoperative neuromonitoring is routinely employed. Patients are monitored closely postoperatively for signs of SCI. If SCI is suspected, a spinal drain is placed emergently, and the CSF pressure is maintained at $<8-12 \mathrm{mmHg}$, whilst MAP is raised above $90 \mathrm{mmHg}$ and beyond until function recovers.

\section{Risks of CSF drainage}

The risks associated with the placement of spinal drains are not negligible, with a pooled overall risk of mild, moderate and severe complications of $6.5-8.1 \%(9,10)$. The mild complications occur in $2-3.9 \%$ of patients following placement of a spinal drain and include dislodged and occluded catheters, mild bleeding, hypotension and leakage of CSF that does not require intervention $(9,10)$. Moderate complications have an incidence of $3.7-4 \%$ and include spinal headache, leakage of CSF requiring blood patch placement and fractured catheter tips requiring operative retrieval $(9,10)$. Severe complications associated with spinal drain placement have an incidence of $2.5-4.2 \%$ and include epidural hematoma, intracranial hemorrhage, neurologic deficits, herniation and infectious processes including meningitis. In addition, a pooled mortality rate of $0.9 \%$ has been cited (9). These complications are certainly not insignificant and can lead to delays in operative intervention and thus inferior patient outcomes due to interruptions in care.

\section{A suggested algorithm for spinal drain placement}

Given the risks associated with placement of CSF drains and the many patients who have not required the drain that was preemptively placed, we suggest preoperative CSF drains be placed only in patients who present with an initial concern for SCI prior to undergoing TEVAR and in those patients who have undergone prior aortic operations. All other patient should not undergo preemptive drain placement. Intraoperative adjuncts to quell the risk of SCI should be maintained. Those patients without spinal drains should be monitored closely intraoperatively and postoperatively with frequent, serial neurologic exams. If SCI is suspected, a spinal drain should be placed emergently and CSF should be maintained at a pressure of $<8-12 \mathrm{mmHg}$. In addition, permissive hypertension should be employed with a target MAP of $>90 \mathrm{mmHg}$, at a minimum, or higher if an initial return of neurologic function is not observed.
Serial neurologic exams should be continued until return of function occurs.

\section{Conclusions}

Though CSF drainage using spinal drains serves an important role in the prevention of SCI following TEVAR, the placement of these drains is not required in every patient. The most effective method for minimizing the incidence of SCI following TEVAR is the use of protocols that implement multiple intraoperative and postoperative adjuncts that contribute to reducing the incidence. Spinal drain placement is not a benign procedure and does carry rare but potentially devastating risks. The other perioperative adjuncts do not impart the additional risks associated with the placement and maintenance of spinal drains. Though the placement of preoperative spinal drains is not mandatory in every patient prior to undergoing TEVAR, facilities performing these operations should have the capability to emergently place spinal drains in the setting of immediate or delayed onset postoperative paraparesis and paraplegia. In addition, patients who are known to be at increased risk for SCI, such as those who have had prior aortic operations, should have preoperative drains placed.

\section{Acknowledgments}

Funding: None.

\section{Footnote}

Conflicts of Interest: The authors have no conflicts of interest to declare.

Open Access Statement: This is an Open Access article distributed in accordance with the Creative Commons Attribution-NonCommercial-NoDerivs 4.0 International License (CC BY-NC-ND 4.0), which permits the noncommercial replication and distribution of the article with the strict proviso that no changes or edits are made and the original work is properly cited (including links to both the formal publication through the relevant DOI and the license). See: https://creativecommons.org/licenses/by-nc-nd/4.0/.

\section{References}

1. Svensson LG, Crawford ES, Hess KR, et al. Experience 
with 1509 patients undergoing thoracoabdominal aortic operations. J Vasc Surg 1993;17:357-68; discussion 368-70.

2. Malloy PC, Raghavan A, Elder T, et al. Cerebrospinal Fluid Drainage During Endovascular Aortic Aneurysm Repair: A Systematic Review of the Literature and Treatment Recommendations. Vasc Endovascular Surg 2020;54:205-13.

3. Bavaria JE, Appoo JJ, Makaroun MS, et al. Endovascular stent grafting versus open surgical repair of descending thoracic aortic aneurysms in low-risk patients: a multicenter comparative trial. J Thorac Cardiovasc Surg 2007;133:369-77.

4. Acher C, Acher CW, Marks E, et al. Intraoperative neuroprotective interventions prevent spinal cord ischemia and injury in thoracic endovascular aortic repair. J Vasc Surg 2016;63:1458-65.

5. Suarez-Pierre A, Zhou X, Gonzalez JE, et al. Association of preoperative spinal drain placement with spinal cord ischemia among patients undergoing thoracic and thoracoabdominal endovascular aortic repair. J Vasc Surg
2019;70:393-403.

6. Miranda V, Sousa J, Mansilha A. Spinal cord injury in endovascular thoracoabdominal aortic aneurysm repair: prevalence, risk factors and preventive strategies. Int Angiol 2018;37:112-26.

7. Estrera AL, Sheinbaum R, Miller CC, et al. Cerebrospinal fluid drainage during thoracic aortic repair: safety and current management. Ann Thorac Surg 2009;88:9-15; discussion 15 .

8. Scali ST, Kim M, Kubilis P, et al. Implementation of a bundled protocol significantly reduces risk of spinal cord ischemia after branched or fenestrated endovascular aortic repair. J Vasc Surg 2018;67:409-423.e4.

9. Rong LQ, Kamel MK, Rahouma M, et al. Cerebrospinalfluid drain-related complications in patients undergoing open and endovascular repairs of thoracic and thoracoabdominal aortic pathologies: a systematic review and meta-analysis. Br J Anaesth 2018;120:904-13.

10. Plotkin A, Han SM, Weaver FA, et al. Complications associated with lumbar drain placement for endovascular aortic repair. J Vasc Surg 2021;73:1513-1524.e2.

Cite this article as: Kemp C, Ikeno Y, Aftab M, Reece TB. Cerebrospinal fluid drainage in thoracic endovascular aortic repair: mandatory access but tailored placement. Ann Cardiothorac Surg 2022;11(1):53-55. doi: 10.21037/acs-2021taes-12 